CORRIGENDUM

doi:10.1038/nature04362

\section{A network-based analysis of systemic inflammation in humans}

Steve E. Calvano, Wenzhong Xiao, Daniel R. Richards, Ramon M. Felciano, Henry V. Baker, Raymond J. Cho,

Richard O. Chen, Bernard H. Brownstein, J. Perren Cobb, S. Kevin Tschoeke, Carol Miller-Graziano, Lyle L. Moldawer, Michael N. Mindrinos, Ronald W. Davis, Ronald G. Tompkins, Stephen F. Lowry \& the Inflammation and Host Response to Injury Large Scale Collaborative Research Program $\dagger$

Nature 437, 1032-1037 (2005) doi:10.1038/nature03985

In this Letter, the affiliations of authors participating in the Inflammation and Host Response to Injury Large Scale Collaborative Research Program are incorrectly listed. The renumbered and amended footnote listing is given here.

†Inflammation and Host Response to Injury Large Scale Collaborative Research Program Paul E. Bankey', Timothy R. Billiar², David G. Camp ${ }^{3}$, George Casella ${ }^{4}$, Irshad H. Chaudry ${ }^{5}$, Mashkoor A. Choudhry ${ }^{5}$, Charles Cooper ${ }^{6}$, Asit De ${ }^{1}$, Constance Elson ${ }^{7}$, Bradley Freeman ${ }^{8}$, Richard L. Gamelli ${ }^{9}$, Celeste Campbell-Finnerty ${ }^{10}$, Nicole S. Gibran ${ }^{11}$, Douglas L. Hayden ${ }^{7}$, Brian G. Harbrecht ${ }^{2}$, David N. Herndon ${ }^{10}$, Jureta W. Horton ${ }^{12}$, William J. Hubbard ${ }^{5}$, John L. Hunt ${ }^{13}$, Jeffrey Johnson ${ }^{14}$, Matthew B. Klein ${ }^{15}$, James A. Lederer ${ }^{16}$, Tanya Logvinenko ${ }^{7}$, Ronald V. Maier ${ }^{11}$, John A. Mannick ${ }^{16}$, Philip H. Mason ${ }^{6}$, Bruce A. McKinley ${ }^{17}$, Joseph P. Minei ${ }^{12}$, Ernest E. Moore ${ }^{14}$ Frederick A. Moore ${ }^{17}$, Avery B. Nathens ${ }^{11}$, Grant E. O'Keefe ${ }^{11}$, Laurence G. Rahme ${ }^{18}$, Daniel G. Remick ${ }^{19}$, David A. Schoenfeld ${ }^{7}$, Martin G. Schwacha ${ }^{5}$, Michael B. Shapiro ${ }^{20}$, Geoffrey M. Silver ${ }^{9}$, Richard D. Smith ${ }^{3}$, John D. Storey ${ }^{21}$, Mehmet Toner ${ }^{22}$, H. Shaw Warren ${ }^{23} \&$ Michael A. West ${ }^{20}$

Affiliations for participants: ${ }^{1}$ Department of Surgery, University of Rochester School of Medicine, Rochester, New York 14642, USA. ${ }^{2}$ Department of Surgery, University of Pittsburgh School of Medicine, Pittsburgh, Pennsylvania 15213, USA. ${ }^{3}$ Pacific Northwest National Laboratory, Richland, Washington 99352, USA. ${ }^{4}$ Department of Statistics, University of Florida, Gainesville, Florida 32611, USA. ${ }^{5}$ Department of Surgery, University of Alabama School of Medicine, Birmingham, Alabama 35294, USA. ${ }^{6}$ Department of Molecular Biology, Massachusetts General Hospital, Harvard Medical School, Cambridge, Massachusetts 02139, USA. 'Department of Biostatistics, Massachusetts General Hospital, Harvard Medical School, Boston, Massachusetts 02114, USA. ${ }^{8}$ Department of Surgery, Washington University School of Medicine, St. Louis, Missouri 63110, USA. ${ }^{9}$ Department of Surgery, Loyola University Stritch School of Medicine, Maywood, Illinois 60153, USA. ${ }^{10}$ Department of Surgery, University of Texas Medical Branch, Shriners Burns Hospital, Galveston, Texas 77550, USA. " Department of Surgery, University of Washington Harborview Medical Center, Seattle, Washington 98104, USA. ${ }^{12}$ Department of Surgery, University of Texas Southwestern Medical Center, Dallas, Texas 75390, USA. ${ }^{13}$ Division of Trauma, Burns, and Critical Care, University of Texas Southwestern Medical Center, Dallas, Texas 75390, USA ${ }^{14}$ Department of Surgery, University of Colorado Denver Health Medical Center, Denver, Colorado 80204, USA. ${ }^{15}$ Burn Center and Division of Plastic Surgery, University of Washington Harborview Medical Center, Seattle, Washington 98104 USA. ${ }^{16}$ Department of Surgery, Brigham and Women's Hospital, Harvard Medical School, Boston, Massachusetts 02115, USA. ${ }^{17}$ Department of Surgery, University of Texas Houston Health Science Center, Houston Medical School, Houston, Texas 77030, USA. ${ }^{18}$ Department of Molecular Biology, Massachusetts General Hospital, Harvard Medical School, Boston, Massachusetts 02114, USA. ${ }^{19}$ Department of Medical Science, University of Michigan Medical School, Ann Arbor, Michigan 48109, USA. ${ }^{20}$ Department of Surgery, Northwestern University Medical School, Chicago, Illinois 60611, USA. ${ }^{21}$ Department of Biostatistics, University of Washington, Seattle, Washington 98195, USA. ${ }^{22}$ Center for Engineering in Medicine, Massachusetts General Hospital, Harvard Medical School, Boston, Massachusetts 02114, USA

${ }^{23}$ Department of Medicine, Massachusetts General Hospital, Harvard Medical School, Boston, Massachusetts 02129, USA
CORRIGENDUM

\section{doi:10.1038/nature04363}

\section{DNA sequence and analysis of human chromosome 18}

Chad Nusbaum, Michael C. Zody, Mark L. Borowsky, Michael Kamal, Chinnappa D. Kodira, Todd D. Taylor, Charles A. Whittaker, Jean L. Chang, Christina A. Cuomo, Ken Dewar, Michael G. FitzGerald, Xiaoping Yang, Amr Abouelleil, Nicole R. Allen, Scott Anderson, Toby Bloom, Boris Bugalter, Jonathan Butler, April Cook, David DeCaprio, Reinhard Engels, Manuel Garber, Andreas Gnirke, Nabil Hafez, Jennifer L. Hall, Catherine Hosage Norman, Takehiko Itoh, David B. Jaffe, Yoko Kuroki, Jessica Lehoczky, Annie Lui, Pendexter Macdonald, Evan Mauceli, Tarjei S. Mikkelsen, Jerome W. Naylor, Robert Nicol, Cindy Nguyen, Hideki Noguchi, Sinéad B. O'Leary, Keith O'Neill, Bruno Piqani, Cherylyn L. Smith, Jessica A. Talamas, Kerri Topham, Yasushi Totoki, Atsushi Toyoda, Hester M. Wain, Sarah K. Young, Qiandong Zeng, Andrew R. Zimmer, Asao Fujiyama, Masahira Hattori, Bruce W. Birren, Yoshiyuki Sakaki \& Eric S. Lander

\section{Nature 437, 551-555 (2005) doi:10.1038/nature03983}

The name of Keith O'Neill was accidentally omitted from the published author list. He is at the first affiliation in the address list.

\section{ERRATUM}

\section{doi:10.1038/nature04361}

\section{Astronomical pacing of methane release in the Early Jurassic period}

David B. Kemp, Angela L. Coe, Anthony S. Cohen

\& Lorenz Schwark

Nature 437, 396-399 (2005)

In the labelling of Fig. 1 of this Letter, the spelling of 'D. semicelatum' was accidentally reversed to read ' $D$. mutalecimes'. It appears correctly in the text. 